\title{
SARS-CoV-2 and Cystic Fibrosis: Expectations Versus Reality, a Literature Review
}

\author{
Authors: \\ * Maria Anna Bantounou, Plascevic Josip \\ School of Medicine and Dentistry, University of Aberdeen, UK \\ *Correspondence to m.bantounou.20@abdn.ac.uk \\ Disclosure: $\quad$ The authors have declared no conflicts of interest. \\ Received: $\quad 02.01 .21$ \\ Accepted: $\quad 09.04 .21$ \\ Keywords: $\quad$ Angiotensin-converting enzyme-2 (ACE2), azithromycin, corticosteroids, COVID-19, \\ cystic fibrosis (CF), cystic fibrosis transmembrane conductance regulator (CFTR), \\ dornase alfa, epidemiology, SARS-CoV-2, TMPRSS2. \\ Citation: \\ EMJ Respir. 2021; DOI/10.33590/emjrespir/20-00275
}

\section{Abstract}

Severe acute respiratory syndrome coronavirus 2 (SARS-CoV-2), responsible for the COVID-19 pandemic, is expected to cause severe illness in people with cystic fibrosis (CF). The infection prevalence and clinical outcomes of this patient cohort to SARS-CoV-2 were explored, alongside contributing factors to the observed response.

Search terms were entered into Medline/PubMed and Embase databases, with relevant published papers written in English chosen.

The COVID-19 trajectory in people with CF (including children) was similar to the general population. Specifically, in Veneto, Italy, the infection rate of people with CF was nearly half compared to the general population (0.19\% versus $0.40 \%$, respectively). Similarly, in Spain, the cumulative incidence of COVID-19 was lower compared to the general population: 32/10,000 and 49/10,000 respectively. Likewise, in Belgium 2.7\% of patients with CF had SARS-CoV-2 antibodies compared with $4.3 \%$ of the general population. Moreover, in Europe, fewer CF-COVID-19 cases and deaths were reported compared to the general population (1.1\%, $0.9 \%$; and $3.2 \%, 2.3 \%$, respectively). Overall, worse outcomes in CF were associated with poorer lung function and post-transplant status.

The encouraging response of people with CF to COVID-19 is hypothesised as due to higher levels of anti-inflammatory angiotensin-1-7 and lower levels of pro-inflammatory IL-6 and protease transmembrane serine protease-2, utilised by SARS-CoV-2 to penetrate cells. Additionally, the use of CF medication, chiefly Dornase alfa and CF transmembrane conductance regulator modulators as well as CF cohort characteristics, predominantly younger age, and early isolation might have mitigated COVID-19 severity.

Thus, people with CF do not appear to have a higher COVID-19 infection prevalence or worse clinical outcomes compared to the general population. 


\section{INTRODUCTION}

Cystic fibrosis (CF) is a chronically deteriorating lung condition that can affect multiple systems, frequently accompanied by a plethora of comorbidities. ${ }^{1}$ Viral infections account for approximately $60 \%$ of CF exacerbations, and people with CF have increased morbidity when exposed to viruses, such as the influenza A virus (H1N1), 2,3 This can be because of the limited antiviral response of airway epithelial cells and co-infection with other bacteria known to affect patients with CF, such as Pseudomonas aeruginosa. ${ }^{3}$ Severe acute respiratory syndrome coronavirus 2 (SARS-CoV-2), the highly contagious virus responsible for the COVID-19 pandemic, leads to worse clinical outcomes in individuals with comorbidities compared to healthy individuals. ${ }^{2}$ People with CF are reported to be at a higher risk of displaying more severe COVID-19 symptoms. ${ }^{4}$ Infection by SARS-CoV-2 in severe cases can cause acute respiratory distress syndrome (ARDS) and even death. ${ }^{3}$

People with CF are assumed at increased risk of worse outcomes when infected by SARS-CoV-2 compared to healthy individuals. This is because of their chronically deteriorating lung function and susceptibility to viral infections, as well as the underlying pathophysiology of their condition and pro-inflammatory and respiratory nature of SARS-CoV-2.2-6 Thus, this cohort is advised to 'shield' by self-isolating.

UK guidelines currently highlight that people with CF may be at increased risk of rapid deterioration if infected by SARS-CoV-2. It is recommended that they should manage their condition as previously instructed, including pharmacological and non-pharmacological interventions, with the additional advice to contact their CF team if experiencing COVID-19 symptoms. ${ }^{4,7}$ However, emerging data suggest that people with CF may not be at a higher risk of SARS-CoV-2 infection or worse clinical outcomes when infected.?

The primary aim of this literature review was to establish the susceptibility of people with CF to SARS-CoV-2 and the prevalence and severity of COVID-19 in this patient cohort. The secondary aim was to identify potential factors that could have contributed to the observed response to SARS-CoV-2, focusing on cellular processes, medication, and cohort characteristics in people with CF.

\section{METHODS}

This literature review was undertaken from $1^{\text {st }}$ November 2020 until 12 $2^{\text {th }}$ February 2021, using a combination of the following search terms: "cystic fibrosis," "SARS-CoV-2," "COVID-19," "ACE-2," "CFTR," "azithromycin," "Dornase-alfa," and "corticosteroids" on the EMBASE and MEDLINE/ PubMed databases. Papers were also identified via the reference lists of examined papers. Only published papers and papers written in English were reviewed and chosen according to content relevance.

\section{RESULTS AND DISCUSSION}

\section{Cystic Fibrosis and Severe Acute Respiratory Syndrome Coronavirus 2: Epidemiology}

Even though data are still preliminary, the emerging picture is that patients with CF experience milder COVID-19 symptoms than expected. A study reported that from 100,000 people infected with COVID-19 (>100 people also having (F), approximately $90 \%$ of people with CF experienced a milder infection. ${ }^{7}$

Furthermore, a retrospective study of 532 people with CF in Veneto, Italy, tested 118 (22.2\%) of these patients (throat and nose swab) who were symptomatic for COVID-19. They identified one patient who had mild symptoms as COVID-19 positive, resulting in an infection rate of $1 / 532$ (0.19\%). Interestingly, the infection rate for people with CF was nearly half compared with the general population. Of the 4,907,704 people in Veneto, 465,433 (9.5\%) were tested, and 19,729 were COVID-19 positive, resulting in an infection rate of 19,729/4,907,704 (0.4\%). ${ }^{2}$ Similarly, a COVID-19-CF observational retrospective survey was conducted by the Spanish CF Society. It demonstrated that the cumulative incidence of COVID-19 in patients with CF was lower compared to the general population: $32 / 10,000$ (0.32\%) and 49/10,000 (0.49\%), respectively. From the 2,498 patients that were registered with the Spanish CF Society (data from 2018), 8/2,498 (0.3\%) COVID-19-positive people were identified, and no fatalities were reported. ${ }^{5}$

Furthermore, a prospective study examined the Belgian SARS-CoV-2 seroprevalence in CF by recruiting 149 patients with CF. Blood tests 
were undertaken to establish the presence or absence of SARS-CoV-2 IgG and IgM antibodies. Positive serologies were reported in $2.7 \%$ (4/149) of patients with CF (3 patients for IgG and 1 for $\mathrm{IgM}$ ) compared with $4.3 \%$ of the Belgian population at the time. A total of $36 / 149$ (24\%) patients had symptoms that were aligned with COVID-19. Six patients required medical attention and were PCR tested for COVID-19, five of whom were hospitalised. Only $1 / 6$ patients tested positive for COVID-19, who paradoxically did not test positive for anti-SARS-CoV-2 antibodies. ${ }^{8}$

Moreover, out of 49,886 registered patients with CF$^{9}$ (from 38 European countries), 554/49,886 (1.1\%) have been COVID-19 positive, 13/49,886 $(2.3 \%)$ of whom needed intensive care, and $5 / 49,886$ (0.9\%) died. ${ }^{10}$ These data align with the response of the general population in Europe. At the time of writing this review ( $1^{\text {st }}$ January 2021), it is estimated that $747,863,079$ people live in Europe. Out of the aforementioned population, 23,844,011 (3.2\%) people were reported to be COVID-19 positive, including 547,196 (2.3\%) people who died. ${ }^{11,12}$

Likewise, a multinational study, with Australia, Canada, France, Ireland, Netherlands, New Zealand, UK, and USA being the participating countries, reported a similar trajectory of COVID-19 infection in people with CF to the general population. Specifically, 40 people with CF who tested positive with COVID-19-were identified with a median age of 33 years (15-59 years). A total of $31 / 40$ (77.5\%) of these people were symptomatic with COVID-19, 28/40 (70\%) experience recurrent bacterial infections, and $11 / 40$ (27.5\%) had received a lung transplant. Furthermore, 25/40 (62.5\%) people were treated with antibiotics, $13 / 40$ (33\%) needed oxygen supplementation, 1/40 (2.5\%) invasive ventilation, and 4/40 (10\%) intensive care unit (ICU) admission. From the 14/40 (35\%) people who were on CF transmembrane conductance regulator (CFTR) modulator therapy, one required ICU admission. No deaths were reported in this cohort.

Severity of the COVID-19 infection between patients with CF and the general population seemed to correlate. A study conducted by the Global Registry Harmonization Group (GRHG) recruited 181 patients with CF from 19 countries.
They identified that people with CF had similar clinical outcomes to the proportion of patients who experienced a worse COVID-19 infection, with worse outcomes associated with poorer lung function and a post-transplant status. The patients' outcomes were analysed according to their transplant status; 141 patients being non-transplant and 27 were post-transplant, with relevant data missing for 13 patients. Overall, non-transplant patients had a significantly lower percentage of admissions compared to the post-transplant patients; 66/141 (66\%) and 20/27 (74\%), respectively. Likewise, fewer nontransplant patients (4\%) were admitted to ICU compared to post-transplant patients (25\%). Overall, seven patients out of the entire cohort died, four of whom were non-transplant. ${ }^{13}$

Similar findings have been reported in paediatric studies. An observational study conducted by the GRHG recruited 105 children with confirmed COVID-19 from 13 countries. The study dataset contained missing values, which were attributed to variability in data collection. The median age of the patient cohort was 10 years (6-15 years), with 31 patients treated with long-term azithromycin, 50 with a CFTR modulator, and 2 being posttransplant. A total of 26/89 (29\%) patients had no COVID-19 symptoms. In symptomatic patients, fever $(46 / 63,73 \%)$ and a change in cough $(38 / 53,72 \%)$ were most reported. A total of $24 / 82$ (29\%) patients were admitted to hospital, with 6/21 (29\%) requiring additional oxygen, 2/20 (10\%) non-invasive ventilation, and $1 / 20(5 \%)$ invasive ventilation. Significantly fewer patients who were on CFTR modulator therapy required hospitalisation compared to patients who were not on such therapy; $6 / 40$ (15\%) and 34/40 (85\%), respectively. No deaths in this patient cohort were attributed directly to COVID-19. One patient, however, died 6 weeks after testing positive for COVID-19, due to disease exacerbation. The authors concluded that children with CF experience a similar COVID-19 disease course compared to children without CF. ${ }^{14}$

Likewise, the expert panel from the European Respiratory Society (ERS) examined the response of children with chronic respiratory conditions to COVID-19 by collating data from 174 ERS centres. Fourteen children with CF were reported to have COVID-19. Due to the incomplete data, one child had to be excluded from the analysis. Severity of 
symptoms varied: 1 child presented with fever; 2 with pneumonia; 4 had a pulmonary exacerbation; and 5 presented with upper respiratory tract infection. From $7 / 13$ (53.8\%) hospitalised children, 3 were admitted to the paediatric ICU, 2 required oxygen, and 1 invasive ventilation. Additionally, 3 children required antibiotics, 3 azithromycin for COVID-19 treatment, and 1 hydroxychloroquine. No deaths were reported in this cohort. The authors of the study concluded that children with CF are at no higher risk of having worse outcomes with COVID-19 compared with the general population. ${ }^{15}$

Therefore, current epidemiological data suggest that people with CF do not have higher infection rate or suffer from worse COVID-19 clinical outcomes compared to the general population.

\section{Cystic Fibrosis and Severe Acute Respiratory Syndrome Coronavirus 2: Cellular Level}

\section{Severe Acute Respiratory Syndrome Coronavirus 2}

Coronaviridae is the viral family of SARS-CoV-2, which is one of the seven types of coronaviruses that infect humans. SARS-CoV and Middle East respiratory syndrome coronavirus also belong to the same viral family and infect the airways, causing respiratory illness. SARS-CoV-2 has a $31 \mathrm{~kb}$ RNA enclosed within a glycoprotein envelope. Spike proteins, Type 1 glycoproteins, are attached and protrude from the envelope. These are broken down by host cell-membrane proteases to facilitate viral entry, chiefly angiotensin-converting enzyme-2 (ACE2) for SARS-CoV-2 because of a single mutation in the spike protein that improves the attachment to ACE2. Cathepsins human airway trypsin-like protease and transmembrane protease serine 2 (TMPRSS2) also contribute to viral entry. After host cells are infected, cytokines are released into the bloodstream causing systemic inflammation, acute lung injury, ARDS, and even death.,16 However, like most human genes, the ACE2 gene also shows polymorphism, which might affect an individual's susceptibility to SARS-CoV-2. ${ }^{3}$

\section{Cystic Fibrosis}

CF is an autosomal-recessive condition and predominantly arises due to a mutation in a gene responsible for encoding the CFTR protein. 17,18 Although several mutation classes have been described, the most common one belongs to Class II, namely Phe508del (previously F508del). ${ }^{19-21}$ The CFTR is a protein channel whose main role involves the regulation of chloride ion balance across the cell membrane. If fully functional, water will be retained in mucus, thereby aiding the process of the mucociliary clearance. However, when the Phe508del mutation occurs, the produced CFTR protein is misfolded and therefore cannot be transported from the endoplasmic reticulum to the cell membrane. ${ }^{22}$ Additionally, the misfolded protein will be recognised and degraded by the ubiquitin-proteasome pathway in a process known as endoplasmic-reticulumassociated degradation..$^{23,24}$ Thus, in the absence of the functional CFTR protein, chloride ions cannot be secreted into the mucus, resulting in an ion imbalance, followed by the retention of viscous mucus and increased susceptibility to infections and inflammation. Collectively, these cellular modulations will lead to typical signs and symptoms of CF. ${ }^{25}$

\section{Cystic Fibrosis and Severe Acute Respiratory Syndrome Coronavirus 2}

It is hypothesised that two key proteins could be involved in pathways to decrease the severity of SARS-CoV-2 infections: ACE2 and TMPRSS2. Specifically, the former cleaves angiotensin I (ANG-1) into ANG-2, and ANG-2 to ANG-1-7. The two enzymatic cleavages produce proteins with contradictory functions: pro-inflammatory ANG-2 and anti-inflammatory ANG-1-7,3,26 ACE2 also supplements the binding of SARS-CoV-2 to the epithelial cells, and TMPRSS2 completes this process, facilitating viral entry into host cells..$^{27,28}$

A recent study utilised previously generated gene microarray data to compare the expression of ACE2 and TMPRSS2 mRNA levels in CF and non-CF airway epithelial cells. ${ }^{29,30}$ In CF cells, ACE2 mRNA levels were higher, while TMPRSS2 levels were decreased. Although a higher ACE2 concentration would enhance the binding of SARS-CoV-2 to host cells, it would also enhance the cleavage rate of ANG-2 to anti-inflammatory ANG-1-7, resulting in milder severity of SARSCoV-2 infection in people with CF. Furthermore, lower TMPRSS2 levels could contribute to the lower infection prevalence in people with CF, as viral entry to the host cells would be hindered. ${ }^{3}$ 
Multiple other CF-associated cellular dysregulations, such as increased autophagy ${ }^{7}$ and lower levels of IL- 6 in the airways of people with CF, might improve their response to SARSCoV-2. Specifically, IL-6 is one of the main cytokines involved in the cytokine storm induced by SARS-CoV-2 and has been associated with worse clinical outcomes. Therefore, lower levels of IL-6 may reduce COVID-19 infection severity. ${ }^{31}$

Further studies are needed to determine whether ACE2, TMPRSS2, and IL-6 levels can be used as clinically useful biomarkers for infection severity or drug targets for COVID-19.

\section{Cystic Fibrosis Drugs and Severe Acute Respiratory Syndrome Coronavirus 2}

The drugs commonly prescribed to people with CF might mitigate the severity of COVID-19 and, indeed, some have been repurposed and are currently in clinical trials to determine their effectiveness against the disease. ${ }^{32,33}$

\section{Corticosteroids}

Low-dose corticosteroids are often prescribed as adjuvant treatment for patients with CF who have not responded to maintenance treatment with oral azithromycin. ${ }^{34}$ Corticosteroids, normally secreted by the adrenal cortex, are shown to be effective in severe COVID-19. ${ }^{32}$ They are not directly antiviral but downregulate pro-inflammatory genes such as $N F K B$ and thus dampen immune and inflammatory response. ${ }^{35}$

In the randomised clinical trial RECOVERY, $6 \mathrm{mg}$ dexamethasone was administered once daily for up to 10 days to $>2,000$ hospitalised patients with COVID-19. Patients on respiratory support had a reduced 28-day mortality, as well as the need for mechanical ventilation and hospitalisation compared to the usual care group. ${ }^{36}$ Other studies also support the protective effect of lowdose or pulsed corticosteroid administration in severe COVID-19,37,38 which is reflected by the recommendation of corticosteroid treatment (including dexamethasone, methylprednisolone, 39 prednisolone, and hydrocortisone) for severe COVID-19 by the World Health Organization (WHO), European Medicines Agency (EMA), National Institutes of Health $(\mathrm{NIH})$, Italian Medicines Agency (AIFA), and Infectious Diseases Society of America (IDSA). ${ }^{32}$
Nevertheless, one systematic review suggested that time for viral clearance, from 8-24 days (standard care group) to 10-29 days (corticosteroid group), infection rate, and antibiotic use are increased with corticosteroid administration. This did not, however, impact length of hospitalisation or mortality. ${ }^{40}$ In contrast, a separate systematic review concluded that corticosteroid treatment was associated with a lengthier hospitalisation. Nonetheless, the corticosteroids were administered to more unwell patients. ${ }^{35}$ Finally, a literature review highlighted that corticosteroids did not improve outcomes in patients with milder COVID-19 infection severity. ${ }^{38}$

Thus, since patients with CF seem to have a milder form of COVID-19, corticosteroids might not offer them any further benefit, as they seem to work best in severe COVID-19 infection.

\section{Dornase Alfa}

The abnormally thick mucus and reduced mucociliary clearance in CF can cause bacteria and noxious particles to get trapped in the airways, leading to infections and chronic inflammation. Neutrophils invade the airways, degranulate and undergo cellular lysis, releasing their DNA, thickening the mucus further, and forming traps for particles and bacteria: the neutrophil extracellular traps (NETs). Dornase alfa, a human recombinant DNase I enzyme used in CF, breaks down NETs, reducing mucus viscosity and improving lung function. ${ }^{41}$

Elevated neutrophil count and NET are also a feature of COVID-19 and a marker for severe respiratory disease. In one study, Dornase alfa (additional to usual care) was administered to three patients with COVID-19, with clinical benefit. This was indicated by the patients' improved oxygen saturation and respiratory rate, as well as reduced coughing, dyspnoea, NET formation, and viral load. Dornase alfa was also shown to lower neutrophil and cytokine levels in blood samples of patients with COVID-19 in vitro, reduce sepsis markers in a septic mouse model in vivo ${ }^{42}$ and even have antiviral properties in vitro in four cell lines infected by SARS-CoV-2.43

Due to the similarities of COVID-19 and CF in neutrophil, cytokine, and NET production, promising preclinical findings, and encouraging case series, ${ }^{44}$ there is a clinical trial investigating 
aerolised intratracheal Dornase alfa as a treatment for SARS-CoV-2-induced ARDS. 33 Therefore, Dornase alfa, which is regularly administered in $\mathrm{CF}^{34}$ could mitigate COVID-19 severity in these patients.

\section{Azithromycin}

Azithromycin, a macrolide antibiotic, is commonly prescribed in CF as antibacterial prophylaxis. ${ }^{34}$ Macrolides inhibit bacterial protein synthesis, neutrophil activation and chemotaxis, and reduce the expression of cell-surface adhesion molecules and pro-inflammatory cytokines. They also increase macrophage activity in the lung alveoli and thus modulate the immune system and inflammatory response. ${ }^{32}$

Additionally, azithromycin has anti-inflammatory and potentially antiviral properties, particularly against respiratory syncytial, influenza, Zika, and Ebola viruses. ${ }^{3,45}$ Its antiviral mechanism is possibly associated with interferon pathways and reduced airway mucus. Alternative hypotheses include prevention of SARS-CoV-2 cellular entry by inhibiting the binding of the COVID-19 spike proteins to $A C E 2^{45}$ or inhibition of the autophagy process by increasing the $\mathrm{pH}$ of key organelles such as endosomes and lysosomes. ${ }^{3}$

The use of azithromycin as a COVID-19 treatment is controversial. ${ }^{46}$ Two French observational clinical studies tested the combination of azithromycin and hydroxychloroquine in patients with COVID-19 and one observational Brazilian clinical study in unconfirmed, symptomatic patients with COVID-19. These studies found that patients treated with the two drugs were rapidly discharged, had significantly more negative nasopharyngeal swabs, and reduced hospitalisations. ${ }^{45}$ Therefore, azithromycin treatment in people with CF may be associated with an improved response against SARS-CoV-2.

Conversely, a prospective study recruited 11 hospitalised patients with severe COVID-19 to evaluate azithromycin and hydroxychloroquine treatment. No clinical benefit of the aforementioned combination was found; one patient died, two required ICU transfer, and one experienced QT interval prolongation within 5 days of treatment. Overall, $8 / 10$ patients were still positive for SARS-CoV-2 on Days 5-6 post-treatment. ${ }^{47}$ Similarly, a retrospective study evaluated 807 patients hospitalised with
COVID-19. Hydroxychloroquine monotherapy or combined with azithromycin did not decrease the risk of death or ventilation and was associated with prolonged hospital admissions. ${ }^{48}$

Furthermore, azithromycin has been associated with cardiac complications, particularly QTC prolongation. ${ }^{32,49} \mathrm{~A}$ study retrospectively reviewed 81 patients treated with hydroxychloroquine and azithromycin and found that $7 / 81$ (8.6\%) had QTC prolongation ( $\geq 500 \mathrm{~ms}$ ), with no patients developing ventricular tachycardia. ${ }^{50}$

Therefore, the AIFA, EMA, U.S. Food and Drug Administration (FDA), and $\mathrm{WHO}$ do not recommend azithromycin or macrolide antibiotics for the treatment of COVID-19 without an underlying bacterial infection. ${ }^{32}$

\section{Cystic Fibrosis Transmembrane Conductance Regulators}

CFTR is critical for bronchodilation and effective mucociliary clearance. In its absence, as in CF, a highly viscous mucus is produced, which triggers an inflammatory response. CFTR modulators repair dysregulated CF cellular processes, increasing airway fluid production, reducing oxidative stress and pro-inflammatory cytokines. ${ }^{7}$

The COVID-19 inflammatory response, particularly when TNF-mediated, reduces the presence of the CFTR protein, primarily in the lungs and brain. This leads to dysregulation of the immune system and physiological changes similar to CF. Specifically, it leads to the activation of cytokines, neutrophils, and macrophages; impediment of mucus clearance and antigen destruction; and promotion of local inflammation and bronchialwall thickening.

Therefore, due to the COVID-19 and CF pathophysiology overlap, there is a rationale for the mechanism of action of CFTR modulators in COVID-19,51 which could also explain the advantageous response of people with CF to COVID-19.

\section{Severe Acute Respiratory Syndrome Coronavirus 2 and Cystic Fibrosis Cohort Characteristics}

Finally, patients with CF might be responding better than expected to SARS-CoV-2 because of cohort characteristics, particularly age and 
habits. People with CF are younger, with the median age in Europe being 18.4 years and in the UK being 21 years, ${ }^{52,53}$ while COVID-19 severity worsens with increasing age., ${ }^{1,54}$ Furthermore, people with CF were instructed to 'shield' earlier in most countries than the general population and for a prolonged time, limiting their exposure to SARS-CoV-2. Finally, people with CF have learned to live with a chronic deteriorating respiratory illness and may have better learned behaviours than the general population regarding personal hygiene and distancing, reducing their risk of infection. ${ }^{1}$

\section{CONCLUSION}

The emerging trend is that people with CF have not had worse clinical outcomes nor a higher infection rate with COVID-19. Multiple hypotheses to explain this exist, including dysregulation of CF cellular processes, certain CF medications, and cohort characteristics of patients with CF. Thus, it would be prudent to collect and analyse up-to-date CF data and update UK guidelines, if appropriate. Other areas of interest for future research are whether Dornase alfa, CFTR modulators, or azithromycin mitigate SARS-CoV-2 infection severity. Additionally, the cellular interplay between CF and SARS-CoV-2 could be investigated further and used to determine an individual's susceptibility to the virus. Lastly, exploration of ACE2, TMPRSS2, and IL- 6 can be undertaken to evaluate if the aforementioned proteins can exist as drug targets or clinically useful biomarkers to predict SARS-CoV-2 infection severity.

\section{References}

1. Cosgriff $R$ et al. A multinational report to characterise SARS-CoV-2 infection in people with cystic fibrosis. J Cyst Fibros. 2020;19(3):355-8.

2. Bezzerri $\vee$ et al. Does cystic fibrosis constitute an advantage in COVID-19 in-fection? Ital J Pediatr. 2020;46(1):143.

3. Stanton BA et al. SARS-CoV-2 (COVID-19) and cystic fibrosis. Am J Physiol Lung Cell Mol Physiol. 2020;319(3):L408-15.

4. BMJ Publishing Group Ltd. BMJ Best Practice. Management of coexisting conditions in the context of COVID-19. 2020. Available at: https://bestpractice. bmj.com/topics/en-gb/3000190/ pdf/3000190/Management\%20of\%20 coexisting $\% 20$ conditions $\% 20$ in $\% 20$ the $\% 20$ context\%20of\%20COVID-19. pdf. Last accessed: 31 December 2020.

5. Mondejar-Lopez $\mathrm{P}$ et al. Impact of SARS-CoV-2 infection in patients with cystic fibrosis in Spain: incidence and results of the national CFCOVID19-Spain survey. Respir Med. 2020;170:106062.

6. Mirza AA et al. Cystic fibrosis and COVID-19: care considerations. Respir Med Case Rep. 2020;31:101226.

7. Peckham D et al. COVID-19 meets cystic fibrosis: for better or worse? Genes Immun. 2020;21(4):260-2.

8. Berardis S et al. SARS-CoV-2 seroprevalence in a Belgian cohort of patients with cystic fibrosis. J Cyst Fibros. 2020;19(6):872-4.

9. European Cystic Fibrosis Society
Patient Registry (ECFSPR). ECFS Patient Registry: Annual Data Report, 2018 data. 2020. Available at: https://www.ecfs.eu/sites/default/ files/general-content-files/workinggroups/ecfs-patient-registry/ ECFSPR_Report_2018_v1.4.pdf. Last accessed: 1 January 2021.

10. European Cystic Fibrosis Society. COVID-CF project in Europe. 2020. Available at: https://www.ecfs.eu/ sites/default/files/general-content files/news/COVID\%20reporting $\% 20$ 12Dec20.pdf. Last accessed: 15 December 2020

11. European Centre for Disease Prevention and Control (ECDC). COVID-19 situation update for the EU/EEA and the UK, as of week 52 2020. 2020. Available at: https:// www.ecdc.europa.eu/en/cases2019-ncov-eueea. Last accessed: 31 December 2020.

12. Worldometer. Europe Population. 2021. Available at: https://www. worldometers.info/world-population/ europe population/\#: :text=The $\% 20$ current\%20population\%20of\%20 Europe,of\%20the\%20total\%20 world\%20population. Last accessed: 1 January 2021.

13. McClenaghan E et al. The global impact of SARS-CoV-2 in 181 people with cystic fibrosis. J Cyst Fibros. 2020;19(6):868-71.

14. Bain $\mathrm{R}$ et al. Clinical characteristics of SARS-CoV-2 infection in children with cystic fibrosis: an international observational study. J Cyst Fibros. 2021:20(1):25-30.

15. Moeller A et al. COVID-19 in children with underlying chronic respiratory diseases: survey results from 174 centres. ERJ Open Res. 2020;6(4):00409-2020.

16. Upadhyay $\mathrm{J}$ et al. Role of inflammatory markers in corona virus disease (COVID-19) patients: a review. Exp Biol Med (Maywood). 2020;245(15):1368-75.

17. Elborn JS. Cystic fibrosis. Lancet 2016;388(10059):2519-31.

18. Klimova B et al. Cystic fibrosis revisited - a review study. Med Chem. 2017;13(2):102-9.

19. Rommens JM et al. Identification of the cystic fibrosis gene: chromosome walking and jumping. Science. 1989;245(4922):1059-65.

20. 20. Riordan JR et al. Identification of the cystic fibrosis gene: cloning and char-acterization of complementary DNA. Science. 1989;245(4922):106673

21. Kerem B et al. Identification of the cystic fibrosis gene: genetic analysis. Sci-ence. 1989;245(4922):1073-80.

22. Dechecchi MC et al. Molecular basis of cystic fibrosis: from bench to bedside. Ann Transl Med. 2018;6(17):334

23. Ward CL et al. Degradation of CFTR by the ubiquitin-proteasome pathway. Cell. 1995;83(1):121-7.

24. Vembar SS, Brodsky JL. One step at a time: endoplasmic reticulumassociated degradation. Nat Rev Mol. 2008;9(12):944-57.

25. Vankeerberghen A et al. The cystic fibrosis transmembrane conductance 
regulator: an intriguing protein with pleiotropic functions. J Cyst Fibros. 2002;1(1):13-29.

26. Riordan JF. Angiotensin-I-converting enzyme and its relatives. Genome Biol. 2003;4(8):225

27. Hoffmann M et al. SARS-CoV-2 cell entry depends on ACE2 and TMPRSS2 and is blocked by a clinically proven protease inhibitor. Cell. 2020;181(2):271-80.e8.

28. Hou $Y$ et al. New insights into genetic susceptibility of COVID-19: an ACE2 and TMPRSS2 polymorphism analysis. BMC Med. 2020;18(1):216.

29. Voisin $\mathrm{G}$ et al. Oxidative stress modulates the expression of genes involved in cell survival in $\triangle \mathrm{F} 508$ cystic fibrosis airway epithelial cells. Physiol Genomics. 2014;46(17):63446.

30. Hampton $\mathrm{TH}$ et al. Does the F508-CFTR mutation induce a proinflammatory response in human airway epithelial cells? Am J Physiol Lung Cell Mol. 2012;303(6):L509-18.

31. Marcinkiewicz J et al. Are patients with lung cystic fibrosis at increased risk for severe and fatal COVID-19? Interleukin 6 as a predictor of COVID-19 outcomes. Pol Arch Intern Med. 2020;130(10):920-1.

32. El Bairi K et al. Repurposing anticancer drugs for the management of COVID-19. Eur J Cancer. 2020;141:40-61.

33. Desilles JP et al. Efficacy and safety of aerosolized intra-tracheal dornase alfa administration in patients with SARS-CoV-2-induced acute respiratory distress syndrome (ARDS): a structured summary of a study protocol for a randomised controlled trial. Trials. 2020;21(1):548.

34. National Institute for Health and Care Excellence (NICE). BNF Treatment summary. Cystic Fibrosis. 2017. Available at: https://bnf.nice.org.uk treatment-summary/cystic-fibrosis. html. Last accessed: 1 January 2021.

35. Budhathoki $P$ et al. Corticosteroids in COVID-19: is it rational? A systematic review and meta-analysis. SN Compr Clin Med. 2020:1-21.

36. The RECOVERY Collaborative Group. Dexamethasone in hospitalized patients with Covid-19. N Engl J Med. 2020;384(8):693-704.

37. Hasan SS et al. Mortality in COVID-19 patients with acute respiratory distress syndrome and corticosteroids use: a systematic review and metaanalysis. Expert Rev Respir Med. 2020;14(11):1149-63.

38. Ahmed MH, Hassan A

Dexamethasone for the treatment of coronavirus disease (COVID-19): a review. SN Compr Clin Med. 2020:110

39. Fadel $\mathrm{R}$ et al. Early short-course corticosteroids in hospitalized patients with COVID-19. Clin Infect Dis. 2020;71(16):2114-20.

40. van Paassen $\mathrm{J}$ et al. Corticosteroid use in COVID-19 patients: a systematic review and meta-analysis on clinical outcomes. Crit Care. 2020;24(1):696.

41. Yang C, Montgomery M. Dornase alfa for cystic fibrosis. Cochrane Database Syst Rev. 2018;9(9):CD001127.

42. Lee $Y Y$ et al. Long-acting nanoparticulate DNase-1 for effective suppression of SARS-CoV2-mediated neutrophil activities and cytokine storm. Biomaterials. 2021;267:120389.

43. Okur HK et al. Preliminary report of in vitro and in vivo effectiveness of dornase alfa on SARS-CoV-2 infection. New Microbes New Infect. 2020;37:100756

44. Weber AG et al. Nebulized inline endotracheal dornase alfa and albuterol administered to mechanically ventilated COVID-19 patients: a case series. Mol Med. 2020;26(1):91.

45. Agrawal A. Azithromycin in coronavirus disease-19: what we know? Open Access Maced J Med Sci. 2020;8(T1):92-6.

46. Ismaila MS et al. Therapeutic options for COVID-19: a quick review. J Chemother. 2021:1-18.

47. Molina JM et al. No evidence of rapid antiviral clearance or clinical benefit with the combination of hydroxychloroquine and azithromycin in patients with severe COVID-19 infection. Med Mal Infect. 2020;50(4):384

48. Magagnoli J et al. Outcomes of hydroxychloroquine usage in united states veterans hospitalized with covid-19. Med (NY). 2020;1(1):114-27. e3

49. Özdemir ïH et al.

Hydroxychloroquine/azithromycin treatment, QT interval and ventricular arrhythmias in hospitalised patients with COVID-19. Int J Clin Pract. 2021;75(2):e13896.

50. Maneikis $\mathrm{K}$ et al. Mitigating arrhythmia risk in Hydroxychloroquine and azithromycin treated COVID-19 patients using arrhythmia risk management plan. Int J Cardiol Heart Vasc. 2021;32:100685.

51. Lidington D, Bolz S. A scientific rationale for using cystic fibrosis transmem-brane conductance regulator therapeutics in COVID-19 patients. Front Physiol. 2020;11:583862.

52. European Cystic Fibrosis Society (ECFS). European Cystic Fibrosis Society annual data report 2013. 2016. Available at: https://www. cysticfibrosis.org.uk/ /media/ documents/the-work-we-do/uk-cfregistry/ecfs-annual-report--what-itmeans-to-the-uk-v7.ashx?la=en. Last ac-cessed: 1 January 2021

53. Cystic Fibrosis Trust. UK Cystic Fibrosis Registry annual data report 2019. 2020. Available at: https:// www.cysticfibrosis.org.uk/ /media/ documents/the-work-we-do/ukcf-registry/registry-report-2019. ashx?la=en. Last accessed: 1 January 2021.

54. Bonanad $\mathrm{C}$ et al. The effect of age on mortality in patients with COVID-19: a meta-analysis with 611,583 subjects. J Am Med Dir Assoc. 2020;21(7):915-8. 